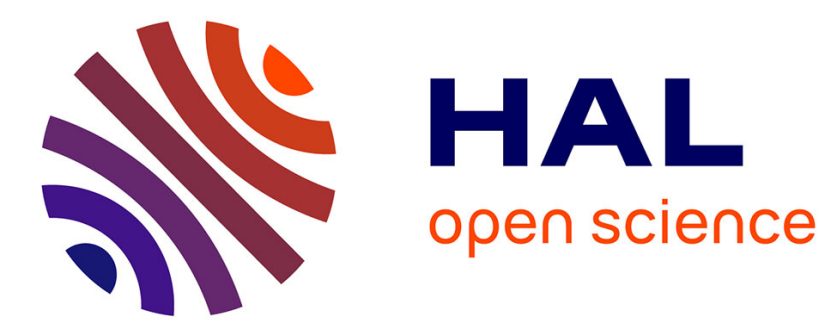

\title{
Quantitative Assessment of the Accuracy of the Poisson-Boltzmann Cell Model for Salty Suspensions
} Yannick Hallez, Joseph Sambasene Diatta, Martine Meireles

\section{To cite this version:}

Yannick Hallez, Joseph Sambasene Diatta, Martine Meireles. Quantitative Assessment of the Accuracy of the Poisson-Boltzmann Cell Model for Salty Suspensions. Langmuir, 2014, vol. 30 ( $\mathrm{n}^{\circ} 23$ ), pp.67216729. 10.1021/la501265k . hal-01250376

\section{HAL Id: hal-01250376 https://hal.science/hal-01250376}

Submitted on 4 Jan 2016

HAL is a multi-disciplinary open access archive for the deposit and dissemination of scientific research documents, whether they are published or not. The documents may come from teaching and research institutions in France or abroad, or from public or private research centers.
L'archive ouverte pluridisciplinaire HAL, est destinée au dépôt et à la diffusion de documents scientifiques de niveau recherche, publiés ou non, émanant des établissements d'enseignement et de recherche français ou étrangers, des laboratoires publics ou privés. 


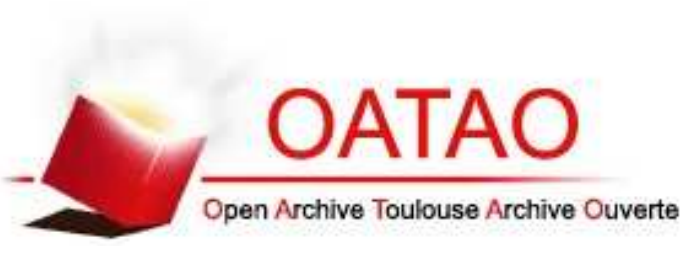

\section{Open Archive TOULOUSE Archive Ouverte (OATAO)}

OATAO is an open access repository that collects the work of Toulouse researchers and makes it freely available over the web where possible.

This is an author-deposited version published in : http://oatao.univ-toulouse.fr/ Eprints ID : 11882

To link to this article : DOI:10.1021/la501265k

URL : http://dx.doi.org/10.1021/la501265k

To cite this version :

Hallez, Yannick and Diatta, Joseph and Meireles, Martine

Quantitative Assessment of the Accuracy of the Poisson-Boltzmann

Cell Model for Salty Suspensions. (2014) Langmuir, vol. 30 ( $\left.\mathrm{n}^{\circ} 23\right)$.

pp. 6721-6729. ISSN 0743-7463

Any correspondance concerning this service should be sent to the repository administrator: staff-oatao@listes-diff.inp-toulouse.fr 


\title{
Quantitative Assessment of the Accuracy of the Poisson-Boltzmann Cell Model for Salty Suspensions
}

\author{
Yannick Hallez,* Joseph Diatta, and Martine Meireles \\ Université de Toulouse, INPT, UPS, Laboratoire de Génie Chimique, 118 Route de Narbonne, F-31062 Toulouse, France \\ CNRS, Laboratoire de Génie Chimique, F-31030 Toulouse, France
}

\begin{abstract}
The cell model is a ubiquitous, fast, and relatively easily implemented model used to estimate the osmotic pressure of a colloidal dispersion. It has been shown to yield accurate approximations of the pressure in dispersions with a low salt content. It is generally accepted that it performs well when long-ranged interactions are involved and the structure of the dispersion is solidlike. The aim of the present work is to determine quantitatively the error committed by assuming the pressure computed with the cell model is the real osmotic pressure of a dispersion. To this end, cell model pressures are compared to a correct estimation of the actual pressures obtained from Poisson-Boltzmann Brownian dynamics simulations including manybody electrostatics and the thermal motion of the colloids. The comparison is performed for various colloidal sizes and charges, salt contents, and volume fractions.

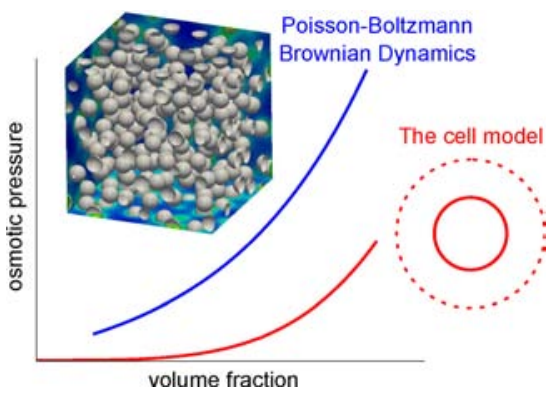
It is demonstrated that the accuracy of the cell model predictions is a function of only the average intercolloid distance scaled by Debye's length $\kappa \bar{d}$ and the normalized colloidal charge. The cell model is accurate for $\kappa \bar{d}<1$ and not reliable for $\kappa \bar{d}>5$ independently of the colloidal charge. In the $1<\kappa \bar{d}<5$ range, covering a wide set of experimental conditions, the colloidal surface charge has a large influence on the error associated with the cell approximation. The results presented in this article should provide a useful reference to determine a priori if the cell model can be expected to predict accurately an equation of state for a given set of physicochemical parameters.
\end{abstract}

\section{INTRODUCTION}

Osmotic pressure is a primary property of a colloidal dispersion. It measures the resistance of a colloidal dispersion to the extraction of the liquid phase in which particles are dispersed. For stabilized colloidal particles, the osmotic pressure increases with the volume fraction of the particles in the dispersion. This equation of state results from many-body interactions on the particle scale. Changes in the state of a dispersion and more practically the crossover to a state where particles start to form aggregates are associated with changes in the equation of state. The prediction of these changes is therefore a key for the practical use of salty colloidal dispersions in many industrial contexts such as filtration, drying, and coating processes. There are a number of experimental methods available to determine the equation of state and the changes of state of colloidal dispersions. ${ }^{1-4}$ Comparing experimental data with the predictions of a model is an excellent way to progress toward a better understanding of the complex physics of such systems. ${ }^{5,6}$ From that perspective, one still needs a clear idea about the accuracy of the retained model.

There are many approaches to predicting the osmotic pressure of a dispersion with computational costs ranging from seconds to thousands of hours. As always, precise methods require daunting numerical efforts whereas fast methods are obtained at the cost of hypotheses with respect to the physics. Among the fast methods, some approaches are based on the prescription of an intercolloid potential of mean force (e.g., Debye-Hückel (DH) or two Yukawa potentials ${ }^{7}$ for moderate surface charges or renormalized $\mathrm{DH}$ potentials for higher surface charges ${ }^{8-10}$ ) and invoke thermodynamic approaches such as solving the Orstein-Zernike equation with a suitable closure relation either analytically ${ }^{7}$ or numerically ${ }^{11}$ to obtain the radial distribution function and then the pressure; other well-established approaches such as the cell model or the renormalized jellium model ${ }^{10,12,13}$ do not require the a priori prescription of such a potential and directly yield the osmotic pressure, although they may have other shortcomings. In the cell model (CM; see refs 14 and15 for a review), the complexity of the multibody electrostatics problem is reduced by assuming that it can be replaced by a one-colloid problem. This approximation stems from the observation that for not too salty and not too dilute suspensions each colloid feels longranged repulsions from its neighbors which induce a solidlike structure in the dispersion. The dispersion is then split into electroneutral cells, with each enclosing a single colloid and the different cells being more or less similar in shape and volume. For simplicity, the geometry of these Wigner-Seitz cells is approximated by another shape matching the geometry of the colloids. Consequently, the model can be solved virtually instantaneously and has the advantage of yielding directly a pressure as a function of the microion density at the cell boundary. ${ }^{16,17}$ The drawback is the impossibility to include 
colloid-colloid interactions or to describe the structure of the dispersion. The osmotic pressure computed with a cell model $\Pi_{\mathrm{CM}}$ can thus be an estimation of only the microionic contribution $\Pi_{\text {micro }}$ to the real osmotic pressure.

In no-salt or low-salt suspensions, the cell model has been shown to provide very good estimates of the osmotic pressure. ${ }^{18-20}$ This is due to the microion contribution to the osmotic pressure being large compared to the pressure contribution generated by the interactions between the colloids. ${ }^{19}$ On the other hand, in salty suspensions the cell model can fail dramatically to provide correct values of the osmotic pressure (refs 11 and 20 and this work). Dobnikar et al. ${ }^{11}$ discussed the consistency between compressibilities computed from both (OCM) simulations based on effective (renormalized) interactions and from the cell model in the charge saturation limit. The renormalization method was based on the cell model itself. They observed empirically that the microionic contribution to the compressibility is consistent with the OCM simulation results when the volume fraction is larger than some function of the salt content and of the saturated effective charge. However, they emphasized the idea that "a better self-consistency is not necessarily synonymous with the fact that $P_{\text {micro }}$ is in itself a better approximation for $P$ ”. The question of the accuracy of the osmotic pressure $\Pi_{\text {micro }}$ approximated by the cell model as an ersatz of the actual osmotic pressure $\Pi$ thus remains open. Moreover, as they chose to focus on the saturated effective charge limit in order to remove one dimensionless parameter from the problem, the influence of the colloidal charge on the accuracy of the cell model approximation remains to be established. In his work, Denton compared the pressure obtained from the cell model to a pressure computed from an effective interactions (EI) model. $^{20}$ The main results confirmed those obtained by Dobnikar et al. ${ }^{11}$ in the sense that for a fixed volume fraction adding salt increases the discrepancy between the cell model and the effective interactions model predictions. The conclusion was that the cell model was "less reliable at intermediate salt concentrations, roughly in the range $0.5 \mathrm{mM}$ $<C_{s}<50 \mathrm{mM}$ ". This observation was reported for a fixed colloidal charge and a fixed range of volume fractions.

Intuitive arguments can be proposed to determine qualitatively if the cell model may be expected to produce an accurate pressure for a given set of physicochemical conditions. A solidlike structure in a dispersion of spherical colloids of radius $a$ can be obtained if long-ranged repulsive intercolloid forces are involved, which requires the Debye length $\kappa^{-1}$ to be larger than some distance representative of half the average intercolloid distance, typically $\bar{d}=a\left(\phi^{-1 / 3}-1\right)$ where $\phi$ is the volume fraction. This qualitative requirement of the interaction range suggests that the cell model should be valid if $\kappa \bar{d}$ is small compared to some $O(1)$ constant. However, the electrostatics problem depends on the three dimensionless parameters $\kappa a, \phi$, and $Z l_{\mathrm{B}} / a$, where $Z$ is the valence of the colloids and $l_{\mathrm{B}}=e^{2} /$ $(4 \pi \epsilon k T)$ is the Bjerrum length ( $\epsilon$ denotes the dielectric constant of the solvent and $e$ is the elementary charge). Only the first two parameters are involved in the aforementioned discussion of the interaction range. It might be anticipated that for a prescribed interaction range the solidlike structure assumed by the cell model is more likely to be maintained for a higher colloidal charge since it enhances the electrostatic forces compared to the thermal forces.

This discussion relies on intuitive arguments, which remain to be proven. Besides, a quantification of the error (denoted
$E\left(\kappa a, \phi, Z l_{\mathrm{B}} / a\right)$ committed when invoking the spherical cell approximation has never been attempted. The aim of this article is to provide such a quantification by comparing the values of the osmotic pressures yielded by both the cell model and reference simulations. Since the aim of this work is to establish quantitatively the error induced in the osmotic pressure when invoking the cell assumption, no other source of discrepancy should be introduced. Hence, the reference simulations should include many colloids moving under the influence of both the many-body electrostatic and thermal forces. In particular, we choose not to introduce pairwise additive forces between the colloids (even with the renormalization procedure) in order to avoid any doubt concerning the origin of discrepancies arising between the cell model and simulation results. This can be achieved with the Poisson-Boltzmann Brownian dynamics (PB-BD) method employed in this work. Thermodynamic quantities will be computed from the results of these simulations. The main results presented hereafter permit us to determine a priori if the Poisson-Boltzmann cell model is reliable for a given set of physicochemical parameters, a feature that we hope will prove useful for future experimental studies.

The Poisson-Boltzmann framework is employed throughout this article although the cell approximation can be invoked with more general theories not restricted to the weak coupling limit. Other classical cell models are the modified PoissonBoltzmann (MPB) cell model, ${ }^{21}$ the DFT cell model, ${ }^{22,23}$ and the HNC/MSA cell model. ${ }^{24}$ Since the results reported here were obtained in the Poisson-Boltzmann framework, they are strictly speaking relevant only in the weak coupling limit. A comment on the applicability of the present results to the other cell models is proposed in the Discussion section.

In section 2, the general framework of the PoissonBoltzmann theory is presented. The numerical PB-BD method is described in section 3 . The results are reported in section 4, and the error induced by the spherical cell approximation is finally discussed in section 5 .

\section{POISSON-BOLTZMANN THEORY}

The most accurate method to obtaining the pressure in a colloidal dispersion would be to use Monte Carlo simulations with the primitive model. However, the numerical effort involved to simulate salty suspensions may become very important due to the number of added salt ions. It is now well established that the Poisson-Boltzmann theory gives accurate results in the weak electrostatic coupling limit $\Xi=2 \pi z^{3} l_{\mathrm{B}}^{2} \sigma / e<$ 1 , where $z$ is the valence of the microions and $\sigma$ is the surface charge density. (See refs 25 and 26 for a more detailed discussion.) In the present work, we consider only the case of a 1:1 electrolyte to ensure that this constraint is satisfied for a broad range of physicochemical conditions.

In the electrolyte, the electrostatic potential is the solution of the Poisson-Boltzmann equation for a dispersion in Donnan equilibrium with a salt reservoir

$$
\tilde{\Delta} \tilde{\psi}=\sinh \tilde{\psi}
$$

The tilde symbol denotes a dimensionless quantity throughout the article. The scales involved in the normalization process are summarized in Table 1 . Inside the colloids, the electrostatic potential obeys the Laplace equation

$$
\tilde{\Delta} \tilde{\psi}=0
$$


Table 1. Scales Used to Nondimensionalize Quantities ${ }^{a}$

\begin{tabular}{ll}
\multicolumn{1}{c}{ quantity } & \multicolumn{1}{c}{ scale } \\
length & $\kappa^{-1}=\left[\epsilon k T / 2 n_{0} e^{2}\right]^{1 / 2}$ \\
electrostatic potential & $k T / e$ \\
surface charge density & $\left(2 n_{0} \epsilon k T\right)^{1 / 2}$ \\
stress & $2 n_{0} k T$ \\
force & $\epsilon(k T / e)^{2}$
\end{tabular}

$a_{e}$ is the elementary charge, $k$ is Boltzmann's constant, $T$ is the temperature, $\epsilon$ is the constant permittivity of the solvent, $\epsilon_{\mathrm{p}}$ is the permittivity constant of the colloids, and $n_{0}$ is the salt ion number density where the electrostatic potential vanishes.

Equations 1 and 2 are coupled at the surface of the colloids by the constant surface charge density condition

$$
\left[\frac{\epsilon_{\mathrm{p}}}{\epsilon} \tilde{\nabla} \tilde{\psi}^{\text {in }}-\tilde{\nabla} \tilde{\psi}^{\text {out }}\right] \cdot \mathbf{n}=\tilde{\sigma}
$$

where $\mathbf{n}$ denotes the outward unit vector normal to the colloid surface. The in and out superscripts refer respectively to quantities taken on the surface of the colloid on the solid side and on solvent side.

In the mean-field Poisson-Boltzmann approximation and for a 1:1 electrolyte, the electrostatic free energy for a constant charge boundary condition can be written as ${ }^{27}$

$$
\tilde{A}=\int_{\tilde{S}} \frac{1}{2} \tilde{\sigma} \tilde{\psi} \mathrm{d} \tilde{S}-\int_{\tilde{V}}\left[(\cosh \tilde{\psi}-1)-\frac{1}{2} \tilde{\psi} \sinh \tilde{\psi}\right] \mathrm{d} \tilde{V}
$$

where $\tilde{S}$ and $\tilde{V}$ denote the surface of the colloids and the volume available to the ions, respectively. (There are several forms for the free energy. We have chosen this expression to avoid the explicit appearance of the electric field since obtaining the latter without numerical smearing in the vicinity of the surface of the colloids is a delicate issue.) The osmotic pressure is related to the free energy by ${ }^{8,28}$

$$
\Pi=-\left.\frac{\partial A}{\partial V}\right|_{N_{c}, T}
$$

where $N_{c}$ is the number of colloids. This definition is consistent with the pressure being defined as the negative derivative of the grand potential functional of the nonlinear $\mathrm{PB}$ theory with respect to the volume, evaluated at the equilibrium profile. ${ }^{17}$

The many-body forces exerted on each colloid are required in the numerical method described hereafter. In the mean-field approach, the force exerted on a colloid with surface $S$ is ${ }^{29}$

$$
\mathbf{F}=\oint_{S} \tau \mathbf{n} \mathrm{d} S
$$

where $\tau$ is the excess osmotic stress tensor defined throughout the electrolyte by

$$
\tilde{\tau}=-(\cosh \tilde{\psi}-1) \mathbf{I}+\left(\tilde{\mathbf{E}} \otimes \tilde{\mathbf{E}}-\frac{1}{2} \tilde{\mathbf{E}}^{2} \mathbf{I}\right)
$$

In this last definition, $\mathbf{I}$ is the unit tensor and $\mathbf{E}=-\nabla \psi$ is the electric field.

For a spherical cell of radius $R$, the general relations recalled in this section are reduced to

$$
\left\{\begin{aligned}
\frac{1}{\tilde{r}^{2}} \frac{\partial}{\partial \tilde{r}}\left(\tilde{r}^{2} \frac{\partial \tilde{\psi}}{\partial \tilde{r}}\right) & =\sinh \tilde{\psi} \text { for } \tilde{a} \leq \tilde{r} \leq \tilde{R} \\
\left.\frac{\partial \tilde{\psi}}{\partial \tilde{r}}\right|_{\tilde{r}=\tilde{a}} & =-\tilde{\sigma} \\
\left.\frac{\partial \tilde{\psi}}{\partial \tilde{r}}\right|_{\tilde{r}=\tilde{R}} & =0
\end{aligned}\right.
$$

The first line is the PB equation (eq 1) for a spherically symmetrical problem. The second line stems from the constant charge boundary condition supplemented by the solution of Laplace's equation in the colloid, $\psi(r<a)=$ constant. The last line is Gauss's law applied to the electroneutral cell. This boundary value problem is only one-dimensional but nonlinear. It can be solved numerically quite fast and is rather straightforward to implement. The osmotic pressure in the cell model reads ${ }^{16,17}$

$$
\Pi_{\mathrm{CM}}=2 n_{0} k T(\cosh \tilde{\psi}-1)
$$

\section{POISSON-BOLTZMANN BROWNIAN DYNAMICS: NUMERICAL METHOD}

The present numerical method (termed PB-BD) is based on a Brownian dynamics approach coupled to a full resolution of the many-body Poisson-Boltzmann problem at each time step in order to compute the forces exerted on the colloids. There have been few attempts to perform such simulations. To our knowledge, it was performed only by Fushiki, ${ }^{30}$ who termed it particle-field molecular dynamics, and the Dobnikar and von Grünberg team. ${ }^{11,31-35}$ Both groups used an overset grid technique. In the present work, the coupled problem in eqs $1-3$ is solved with the level-set/ghost-fluid method. ${ }^{36}$ This approach has the advantage of being amenable to solve the problem for nonspherical colloids without any further development. The drawback is that without an adaptive mesh technique the computational cost is higher than that of the overset grid technique. The outlines of the numerical method are presented hereafter.

Initially, $N_{\mathrm{c}}=65$ colloids are placed randomly in a cubic triperiodic box. (To ensure that the results are not affected by finite size effects, a few simulations were also carried out with $N_{\mathrm{c}}=28$ and 217. The free energy per colloid was the same for $N_{c}=65$ and 217. Note that Linse and Lobaskin ${ }^{25}$ reported that $N_{c} \geq 40$ was sufficient in their simulations presented in refs 37 and 25 , in line with the present verification.) Their positions are evolved in time with the classical Ermak and McCammon scheme. $^{38}$ (More sophisticated BD schemes exist, in particular, to take into account at least partially hydrodynamic interactions between the colloids or electrokinetic effects. ${ }^{39}$ Although the use of the present simple model might slightly alter the pressures obtained in the PB-BD simulations for the highest volume fractions investigated, it is not expected to change the main results of this work significantly.) Possible collisions are treated with the method proposed by Foss and Brady. ${ }^{40,41}$ The external force involved in the $\mathrm{BD}$ displacement is the manybody force (eq 6) whose computation requires the solution of the coupled Poisson-Boltzmann (eq 1) and Laplace (eq 2) equations throughout the numerical domain. This computation is performed at every Brownian time step and is the most CPUexpensive part of the algorithm: the numerical domain containing the colloids and the continuous electrolyte is 
discretized on a $256^{3}$ uniform Cartesian grid (the forces and the free energy are thus obtained with a numerical error of less than $5 \%$ for the range of parameters investigated in this work). The interface between the solid and liquid phases is represented by the zero level of a signed distance function $\Phi$ whose absolute value at any point on the grid is the distance to the closest interface and whose sign is negative inside the colloids and positive in the electrolyte (Figure 1). The PB and Laplace

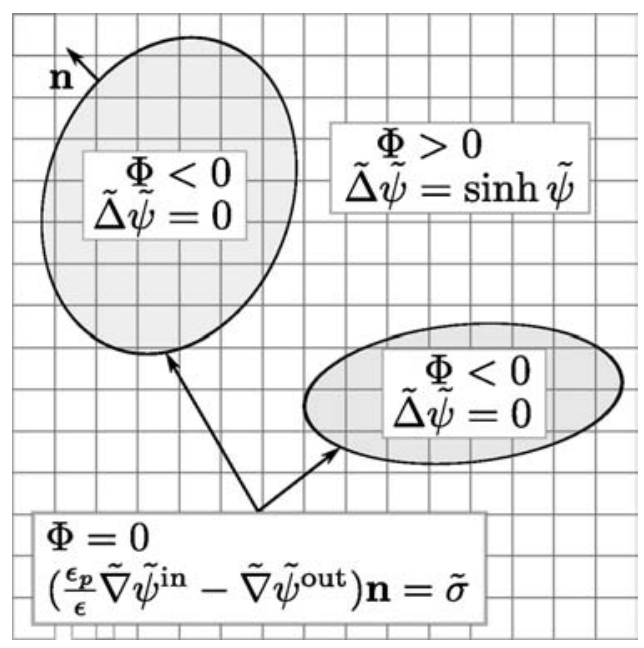

Figure 1. Sketch of the coupled resolution of eqs $1-3$ on a Cartesian grid with the level set/ghost fluid method.

equations are discretized on the grid with standard secondorder finite differences away from the surface of the colloids. Near the interfaces, the discretization of the Laplacian operator is modified to enforce implicitly the boundary condition (eq 3) with the level set/ghost fluid method. ${ }^{36,42,43}$ The complete scheme results in a $256^{3} \times 256^{3}$ sparse, symmetric, and nonlinear system. The latter was solved with an inexact Newton iteration and a conjugate gradient technique with a Jacobi preconditioner for the inner linear systems. This was implemented thanks to the PETSc library. ${ }^{4-46}$

The electrostatic forces are evaluated numerically from relation 6 , in which the integration surface $S$ is shifted from the actual surface of the colloids by a small amount $d$ to avoid numerical inaccuracies. This is possible because the excess osmotic stress tensor is divergence-free in the electrolyte. ${ }^{30,34,47}$ The shifted interface is simply defined by a level set function $\Phi^{\prime}$ $=\Phi-d$ (independently of the geometry of the colloids which prescribes $\Phi)$. The numerical integration of relation 6 on the surface defined by $\Phi^{\prime}$ is then performed using the discrete $\delta$ function approach described in ref 48 .

The free energy is computed from eq 4 , where the surface integral is evaluated with the aforementioned method based on the actual surface of the colloids and the volume integral is performed throughout the entire domain but with the integrand multiplied by a discrete Heaviside function defined using the level set function $\Phi$.

The complete problem solved at each Brownian time step is summarized in Figure 1.

\section{RESULTS}

The results discussed hereafter can be classified into three data sets whose parameters are summarized in Table 2 . The results of data set $A$ are presented on Figures $2(a-c)$ and $3(a-c)$.
Table 2. Physical Parameters Corresponding to the Different Data Sets Discussed in This Article

\begin{tabular}{clll} 
data set & $a(\mathrm{~nm})$ & $Z l_{\mathrm{B}} / a$ & \multicolumn{1}{c}{ source } \\
A & 7.5 & 2.48 & this work \\
B & 50 & 7.3 & ref 20 and this work \\
C & 73.1 & $>1000^{a}$ & ref 11
\end{tabular}

${ }^{a}$ The actual bare charge is very large, its precise value being irrelevant since it corresponds to the saturated effective charge regime, with $Z_{\text {eff }}^{\text {sat }} l_{\mathrm{B}} / a \approx 10$. See ref 11 for more details.

They correspond to spherical colloids with a radius $a=7.5 \mathrm{~nm}$ and a surface charge density $\sigma=0.036 e / \mathrm{nm}^{2}$. The Coulomb coupling parameter is $\Xi \simeq 0.12$, so the PB framework can be safely employed. The ionic strength was increased progressively from 1.558 to 6.439 and $25.758 \mathrm{mM}$, with the salt contents corresponding to $\kappa a=1,2$ and 4, respectively. Data set B corresponds to the parameters of Figure 5(b) in ref 20, i.e., $a=$ $50 \mathrm{~nm}, Z=500$, which yields $\Xi \simeq 0.053$. An example of a PB$\mathrm{BD}$ simulation result corresponding to these parameters is presented in Figures 2(d) and 3(d). The results of data set $C$ will be discussed in the next section.

The nonideal part of the osmotic pressure $\Pi_{\mathrm{NI}}=\Pi-\rho_{\mathrm{c}} k T$ computed with both the Poisson-Boltzmann Brownian dynamics (PB-BD) and the cell model (CM) is reported on Figure 2. Under low-salt conditions, here for $\kappa a=1$, the cell model provides osmotic pressure values in excellent agreement with the PB-BD simulations, as shown in Figure 2(a) and as expected. ${ }^{11,18,20}$ When the salt content is augmented, the cell model predictions increasingly underestimate the correct osmotic pressure as depicted in Figure $2(b-d)$. The underestimation of the osmotic pressure by the cell model was already observed for a dispersion of stiff-chain polyelectrolytes modeled both by many-body MD simulations and cylindrical cell models. ${ }^{49}$ This phenomenon is due to the absence of interactions between the colloids in the cell model. ${ }^{11,19,20,49,50}$ Note that the maximum discrepancy between the cell model and the PB-BD simulations is obtained for the conditions of Figure 2(d), i.e., for the smallest salt concentration in the reservoir! Although it might be obvious to some readers, the term low salt frequently used in the literature to characterize the conditions for which the cell model is accurate should rather be understood as low $\kappa a$ values. Indeed, the conditions of Figure 2(d) correspond to the data obtained for the highest $\kappa a$ value investigated here.

Another interesting observation is the improvement of the cell model predictions as the volume fraction is increased while holding $\kappa a$ fixed. For example, under the conditions of Figure 2 (c) the cell model prediction is only about $5 \%$ of the correct value at $\phi=0.10$ while it is $40 \%$ of the correct value at $\phi=$ 0.22 . For this reason, any criterion aiming to provide a domain of validity of the cell model should involve at least both dimensionless parameters $\kappa a$ and $\phi$. As mentioned in the Introduction, the colloidal charge is also expected to play a role, which will be discussed in the next section.

\section{DISCUSSION OF THE ERROR ASSOCIATED WITH THE CELL APPROXIMATION}

The pressure in a colloidal dispersion can be written in the form

$$
P=\rho_{\mathrm{c}} k T+P_{\mathrm{ocm}}+P_{\text {micro }}
$$




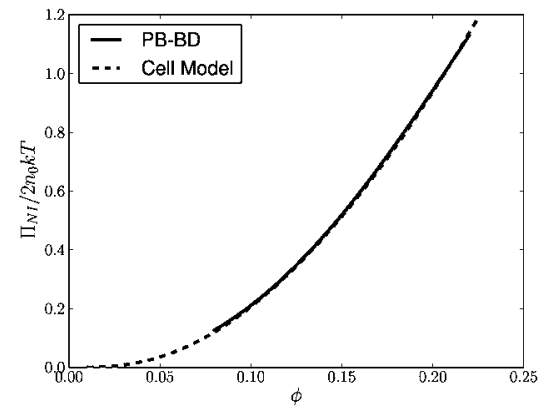

(a) $I=1.558 \mathrm{mM}, \kappa a=1, \tilde{\sigma}=2.52$

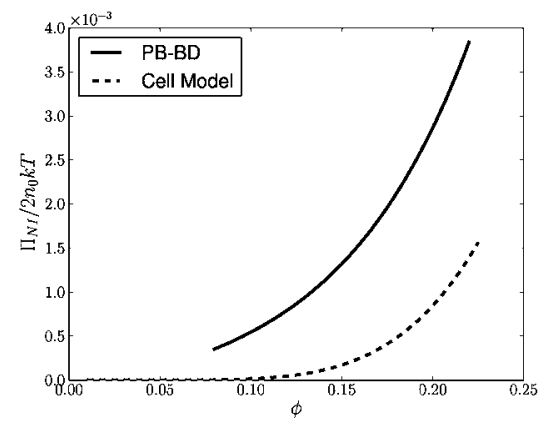

(c) $I=25.758 \mathrm{mM}, \kappa a=4, \tilde{\sigma}=0.62$

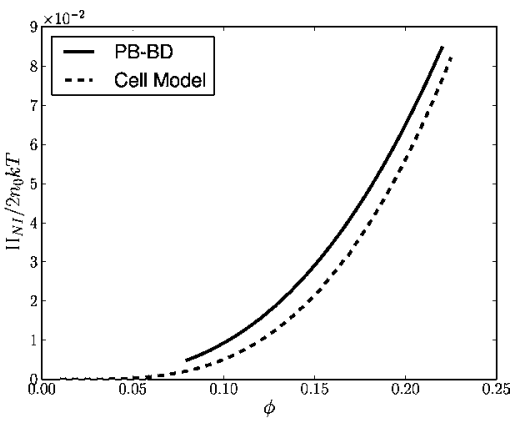

(b) $I=6.439 m M, \kappa a=2, \tilde{\sigma}=1.25$

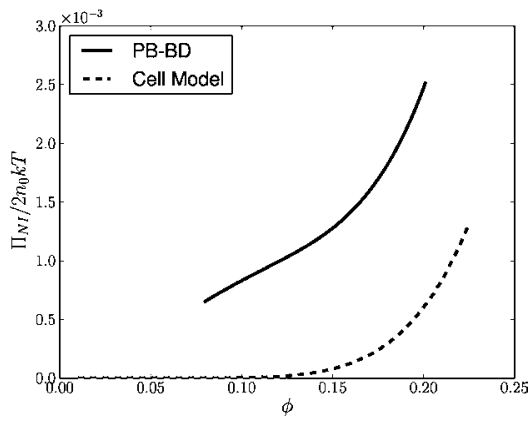

(d) $I=1 \mathrm{mM}, \kappa a=5.254, \tilde{\sigma}=1.389$

Figure 2. Nonideal part of the osmotic pressure computed with the Poisson-Boltzmann Brownian dynamics simulations (PB-BD) and the cell model.

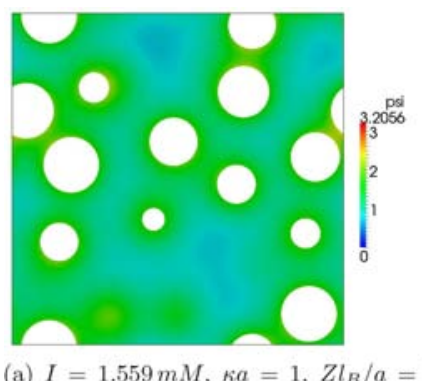

(a) $I=1.559 \mathrm{mM}, \kappa a=1, Z l_{B} / a=(\mathrm{b})$ 2.48

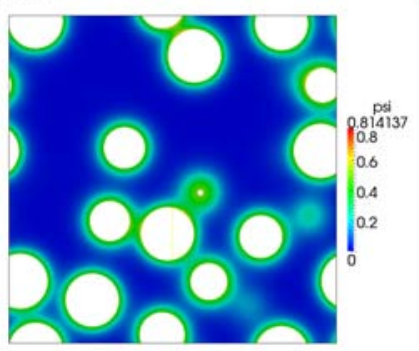

(c) $I=25.758 \mathrm{mM}$, $\kappa a=4, Z l_{B} / a=(\mathrm{d}) I=1 \mathrm{mM}, \kappa a=5.254, Z l_{B} / a=$ 2.48 7.3

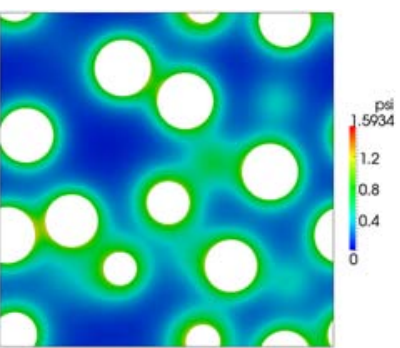

$I=6.439 \mathrm{mM}, \kappa a=2, Z l_{B} / a=$ 2.48

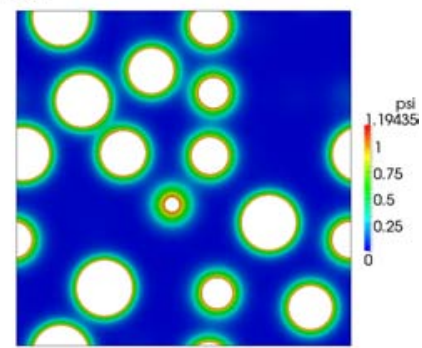

Figure 3. Color map of the dimensionless electrostatic potential $\tilde{\psi}$ in a slice through the domain of $\mathrm{PB}-\mathrm{BD}$ simulations for $\phi=0.16$.

where $P_{\mathrm{ocm}}=1 /(3 \mathrm{~V})\left\langle\sum_{i \in \text { coll }} \mathbf{r}_{i} \mathbf{F}_{i}\right\rangle_{\mathrm{c}}$ is the colloid-colloid virial contribution and $P_{\text {micro }}$ arises from the coupling between colloids and microions. ${ }^{19,51}$ By construction, the cell model can capture only $P_{\text {micro }}$. Therefore, a necessary condition for the cell model to provide a good estimation of the total osmotic pressure is $P_{\text {micro }} \gg P_{\mathrm{ocm}}$. This condition has been observed for low-salt contents. ${ }^{11,19,50}$ As mentioned in the Introduction, if the Debye length is large compared to the typical interparticle distance $(\kappa \bar{d} \ll 1)$, then each colloid feels a net force resulting from the electrostatic potential generated by several neighbors which tends to be rather homogeneous (Figure 3(a)). Without significant potential gradients around the colloid, the global force exerted on it is bound to be small according to definitions in eqs 6 and 7. Meanwhile, since the average interparticle distance $\bar{d}$ is short compared to $\kappa^{-1}$, the electrostatic potential and thus the ion concentration are high at the location of Wigner-Seitz cell boundaries (Figure 3(a)). The contribution $P_{\text {micro }}$ is thus enhanced. On the other hand, under high-salt conditions (for a prescribed volume fraction) $P_{\mathrm{ocm}}$ is a good estimation of the osmotic pressure. ${ }^{11}$ Indeed, when the $\bar{d} \gg \kappa^{-1}$ the positions of the colloids are much more influenced by the Brownian motion and the solidlike structure is lost. The local potential field is thus generated by one or two interfaces only and is not very homogeneous and isotropic (Figures $3(\mathrm{c}, \mathrm{d})$ ). Hence the net force exerted on each colloid is significant while the boundaries of Wigner-Seitz cells lie essentially where the ion concentration and thus $P_{\text {micro }}$ are very low.

This qualitative discussion leads to the conclusion that the cell model should provide reliable results when the average intercolloid distance scaled by the Debye length $\kappa \bar{d}=\kappa a\left(\phi^{-1 / 3}\right.$ -1 ) is small compared to some $O(1)$ constant. To the best of our knowledge, this criterion has never been expressly provided in the literature, and although it might seem intuitive, it remains to be proven. We computed the error associated with the use of the cell model pressure as an ersatz of the real osmotic pressure as a function of parameters $\kappa a, \psi$, and $Z l_{\mathrm{B}} / a$. When the reference osmotic pressure is available from the nonlinear PB$\mathrm{BD}$ simulations, this error is directly computed as

$$
E=\frac{\Pi_{\mathrm{PB}-\mathrm{BD}}-\Pi_{\mathrm{CM}}}{\Pi_{\mathrm{BD}-\mathrm{PB}}}
$$

where $\Pi_{\mathrm{PB}-\mathrm{BD}}$ is the nonideal part of the pressure obtained from the Poisson-Boltzmann Brownian dynamics simulations 
encompassing both $P_{\mathrm{ocm}}$ and $P_{\text {micro }}$ and $\Pi_{\mathrm{CM}}$ denotes the osmotic pressure yielded by the cell model, an estimate of $P_{\text {micro }}$ only. These quantities correspond to those reported on Figure 2. Note that with this definition $E$ is a measure of the relative weight of $P_{\mathrm{ocm}}$ in the total nonideal part of the osmotic pressure. If the reference pressure is obtained from a renormalized one-component model (ROCM), ${ }^{11,20} \Pi_{\mathrm{ROCM}}$ may contain an error associated with the renormalization procedure. In this case, the thermodynamics of a real problem $\left\{\right.$ solve nonlinear electrostatics with real parameters $\left(\kappa a, \phi, Z l_{\mathrm{B}}\right.$ ) a)\} are supposed to match those of an associated linear problem \{solve linearized electrostatics with renormalized parameters $\left.\left(\kappa_{\mathrm{eff}} a, \phi, Z_{\mathrm{eff}} l_{\mathrm{B}} / a\right)\right\}$. Comparing $\Pi_{\mathrm{ROCM}}$ and $\Pi_{\mathrm{CM}}$ would yield an error resulting from both the cell approximation and the renormalization approximation. However, by comparing the osmotic pressure from the associated linear problem $\left(\Pi_{\mathrm{ROCM}}\right)$ and the osmotic pressure of the linearized cell model with renormalized parameters (RLCM), only the spherical cell approximation is tested. Hence, when exploiting data from the ROCM models of refs 20 and 11, the error is computed from

$$
E^{\prime}=\frac{\Pi_{\mathrm{ROCM}}-\Pi_{\mathrm{RLCM}}}{\Pi_{\mathrm{ROCM}}}
$$

where the colloid entropic contribution is also removed from $\Pi_{\text {ROCM}}$. Once again, with this definition the error results are made independent of the details of any renormalization procedure. When considering the data computed by Denton, ${ }^{20}$ $E^{\prime}$ compares the pressure obtained from the variational perturbation theory, based on an effective pair potential computed from the LPB-EI model, to the linear cell model pressure with the same effective parameters. (Therefore, it cannot be used to compare the LPB-EI results to the PB-BD results. This interesting question is beyond the scope of the present article and is left for future work.) The error computed from Dobnikar et al.'s results ${ }^{11}$ was obtained by first calculating pressures from the compressibilities. The latter are issued from the resolution the Ornstein-Zernike equation with the Rogers-Young closure and an effective interaction potential corresponding to a renormalization with the cell model. ${ }^{9}$

The relative error $E$ that would be committed by assuming the cell model pressure to be the actual osmotic pressure is displayed in Figure 4 as a function of the normalized average distance $\kappa \bar{d}=\kappa a\left(\phi^{-1 / 3}-1\right)$. The results obtained from the comparison with the present $\mathrm{PB}-\mathrm{BD}$ results appear as black symbols (continuous lines for $Z l_{\mathrm{B}} / a=2.48$ and dashed lines for $\left.Z l_{\mathrm{B}} / a=7.3\right)$. Those obtained by processing the pressure yielded by the LPB-EI model of Denton ${ }^{20}$ for $Z l_{\mathrm{B}} / a=7.3$ are reported as open circles. The error evaluated from the simulations of Dobnikar et al. ${ }^{11}$ for $Z l_{\mathrm{B}} / a>1000$ are plotted as gray symbols. The latter two data sets correspond to an error defined as $E^{\prime}$ and plotted against the variable $\kappa_{\text {eff }} \bar{d}$ for the reasons detailed previously. There are three main comments to be made about Figure 4.

First, every error curve has the same shape, showing small errors for large interaction ranges $(\kappa \bar{d}<1)$ and very important errors for small interaction ranges corresponding to $\kappa \bar{d}>5$, independently of the colloidal charge. This confirms the general qualitative criterion $\kappa \bar{d}<O(1)$ for the validity of the cell model. It is, however, enlightening to consider the results displayed in Figure 4 in more detail. It appears that for very low values of $\kappa \bar{d}$ the error associated with the spherical cell approximation increases again instead of staying small, as would

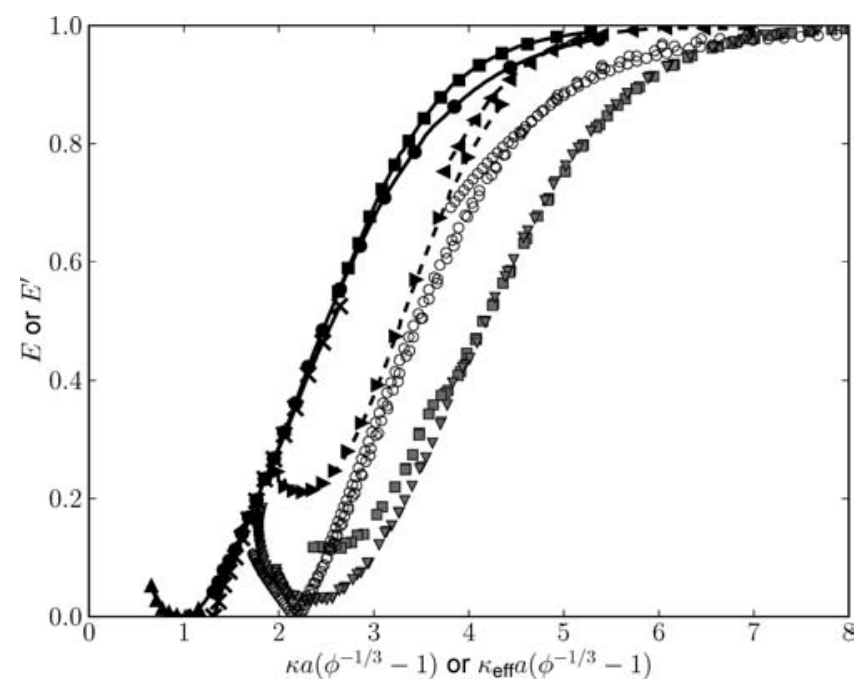

Figure 4. Relative error in the osmotic pressure associated with the spherical cell approximation. Black curves and symbols, by comparing present PB-BD simulations and the cell model, with continuous lines for $Z l_{\mathrm{B}} / a=2.5$ and dashed lines for $Z l_{\mathrm{B}} / a=7.5(\boldsymbol{\square}, \kappa a=4 ; \times, \kappa a=2$; ๑, $\kappa a=2$ and linearized PB equation; $\mathbf{\Delta}, \kappa a=1 ;\langle, \kappa a=5.2 ; \boldsymbol{\rangle}, \kappa a$ $=2.8)$. Open symbols: by comparing the LPB-EI results from ref 20 and LCM results with effective parameters, for $Z l_{\mathrm{B}} / a=7.3$ (the different superimposed curves correspond to $\kappa a=1.6,2.3,3.3$, and 5.2). Gray symbols: by comparing renormalized OCM simulations from ref 11 and the cell model for $Z l_{\mathrm{B}} / a>1000$ (gray $\mathbf{\square}, \kappa a=1.5$; gray $\nabla, \kappa a=0.5)$. For the open and gray symbols, the abscissa is $\kappa_{\text {eff }} a\left(\phi^{-1 / 3}\right.$ $-1)$.

be expected from the intuitive interaction-range argument. This is observed for every data set, precluding the possibility of a numerical artifact. It must be kept in mind that the qualitative discussion presented in the Introduction is relevant only to determining if the pressure is dominated by the microion contribution or by the macroion contribution. However, there is another source of error in the spherical cell model: the geometry of the cell itself. Indeed, even in the very low $\kappa \bar{d}$ limit in which the microionic contribution to the pressure $\Pi_{\text {micro }}$ is virtually equal to the exact pressure, the equality $\Pi_{\mathrm{CM}}=\Pi_{\text {micro }}$ is bound to fail: the maximum packing fraction is 1 in the cell model whereas the dispersion state will change dramatically when the real maximum packing fraction is obtained for $\phi \approx$ 0.6. Even for more moderate volume fractions, the spherical symmetry of the Wigner-Seitz cell might not permit us to compute the electrostatic field correctly, as observed in some preliminary comparisons between the cell model and a numerical implementation of a cubic cell model with the present $3 \mathrm{D} P B$ solver. The small increase in the $\mathrm{CM}$ error presented oin Figure 4 at the lowest $\kappa \bar{d}$ values (highest volume fractions) might be ascribed to this phenomenon.

The second striking observation is the collapse of all of the error data for a given colloidal charge. The errors computed from the $\mathrm{PB}-\mathrm{BD}$ results for $Z l_{\mathrm{B}} / a=2.48$ using either the nonlinear or the linearized $\mathrm{PB}$ equation (black continuous lines) superimpose almost perfectly, like those obtained in the charge-saturation regime $Z l_{\mathrm{B}} / a>1000$ (gray symbols). The error curves yielded by the pressures obtained from the LPB-EI model for $Z l_{\mathrm{B}} / a=7.3$ (open symbols) collapse very well, and the agreement with the PB-BD results for the same colloidal charge is rather good. The shift in the error curves toward higher $\kappa \bar{d}$ values is thus due to the increase in the colloidal 
charge and not to a modeling error. The apparition of a unique master curve for each given surface charge, while data were obtained for various $\kappa a$ and $\phi$ values, indicates that the error $E$ is actually a function of the two dimensionless groups $\kappa a\left(\phi^{-1 / 3}-1\right)$ and $Z l_{\mathrm{B}} / a$ instead of the three dimensionless variables $\kappa a, \phi$, and $Z l_{\mathrm{B}} / a$. This conclusion is beyond the simple idea that the error should be small (respectively large) for small (respectively large) values of $\kappa \bar{d}$.

Up to this point, we have shown that the intuitive criterion determining the domain of validity of the cell model $\kappa a\left(\phi^{-1 / 3}-1\right)<O(1)$ is correct but that the value of the constant depends on the surface charge of the colloids. Assuming a $20 \%$ error in the pressure to be acceptable, the constant is found to lie between 2 and 3 for the different values of $\mathrm{Zl}_{\mathrm{B}}$ / $\mathrm{a}$ tested here (Figure 4 ). For the lowest charges of data set $\mathrm{A}$ and for $\kappa a=2$ and 4 , the linearized $\mathrm{PB}$ equation produced osmotic pressures identical to those determined with the nonlinear PB equation, indicating that the Debye-Hückel $(\mathrm{DH})$ approximation is valid without any renormalization procedure. On the other hand, the results of data set $\mathrm{C}$ were obtained by ensuring that the charge-saturation regime was attained so that the osmotic pressure value becomes independent of any further increase in the surface charge density. In the $\mathrm{DH}$ limit, the analytical solution of the cell model shows that $P_{\text {micro }}$ scales as $Z^{2}$. The $\mathrm{DH}$ pairwise force also scales as $Z^{2}$ so that $P_{\mathrm{ocm}} \approx Z^{2}$. Hence, the error committed by the cell model $E$ defined as eq 11 is independent of $Z$ in the weak charge limit. In the high charge regime reached at saturation of the effective charge, $P_{\text {micro }}$ becomes independent of $Z$, and the $P_{\mathrm{ocm}}$ contribution involves a $\mathrm{DH}$ force renormalized with the effective charge at saturation $Z_{\text {eff }}^{\text {sat }}$ a quantity independent of $Z$. In other words, the error $E$ also becomes independent of $Z$ in the high charge limit. To conclude, it appears that the values 2 and 3 are actually bounds for the constant in the relation $\kappa \bar{d}<O(1)$ determining (partly) the domain of validity of the cell model.

The third and last observation is the strong charge dependence of the error in the range $1<\kappa \bar{d}<5$. As expected from the qualitative arguments presented in the introduction, the error is reduced if the colloidal charge is increased while keeping the interaction range constant (fixed $\kappa \bar{d}$ value). Although the different error curves are relatively close, keeping in mind that the surface charge density varies by several orders of magnitude for the different data sets, this observation is relatively satisfying only concerning the functional form of $E\left(\kappa a\left(\phi^{-1 / 3}-1\right), Z l_{\mathrm{B}} / a\right)$. Unfortunately, Figure 4 also reveals that the transition from the small error to large error regimes is very steep. From a practical point of view, the criterion $\kappa a\left(\phi^{-1 / 3}-1\right)<2-3$ is thus proven to be only qualitative, whereas the plots in Figure 4 provide a quantitative assessment of the accuracy of the calculated pressure. For example, this figure shows that for $\kappa \bar{d}=3$ (e.g., a dispersion with a volume fraction of $6 \%$ colloids of radius $7.5 \mathrm{~nm}$ in contact with a reservoir with $6 \mathrm{mM}$ salt) the cell model error is $75 \%$ for a surface charge density of $\sigma=0.036 e / \mathrm{nm}^{2}, 40 \%$ for $\sigma=0.1 e$ / $\mathrm{nm}^{2}$, and only $10 \%$ in the charge-saturation limit. So in practice, the trust one ought to put in the cell model is very dependent on the colloidal charge in the range $1<\kappa \bar{d}<5$, with the latter being possibly determined by the $\mathrm{pH}$ or the dispersion chemistry.

Since the results reported here were obtained in the Poisson-Boltzmann framework, they are strictly speaking relevant only for systems in the weak coupling limit. If strong electrostatic coupling effects are important, for example, as observed for multivalent counterions, then the interparticle forces are reduced and can even become attractive, possibly leading to a phase separation which cannot be predicted with the PB theory. ${ }^{25}$ In this context, the qualitative idea that the cell model is adequate for repulsive interactions with a range greater than the average intercolloid distance still holds, but the quantitative results of Figure 4 might not be relevant anymore. The results of Figure 4 might thus not be directly extended to the MPB-, DFT-, and HNC/MSA-cell models mentioned in the Introduction. It is, however, expected that the present conclusions can be enlarged to electrostatic couplings larger than those imposed by the use of the PB framework for physicochemical conditions in which the finite size of the ions and the electrostatic correlations between them do not significantly modify the microstructure of the system.

\section{CONCLUSIONS}

The aim of the present article was to determine quantitatively to which extent a fast and rather easily implemented model, namely, the Poisson-Boltzmann cell model, could provide reliable estimations of the osmotic pressure in a colloidal dispersion as a function of the three dimensionless parameters driving the electrostatics problem: $\kappa a, \phi$, and $Z l_{\mathrm{B}} / a$. The error intrinsic to the spherical cell approximation was computed by comparing the associated osmotic pressure to its counterpart obtained from nonlinear Poisson-Boltzmann Brownian dynamics simulations. The latter numerical approach includes all many-body interactions and is free of any modeling hypothesis (in the weak coupling limit, conferring its validity to the Poisson-Boltzmann theory).

Although the error in the cell model $E$ depends a priori on the three variables $\kappa a, \phi$, and $Z l_{\mathrm{B}} / a$, it was shown that $E$ is actually a function of the two dimensionless groups $\kappa \bar{d}=$ $\kappa a\left(\phi^{-1 / 3}-1\right)$ and $Z l_{\mathrm{B}} / a$ only. Assuming that the osmotic pressure can be replaced by its microionic contribution was shown to be always valid for $\kappa \bar{d}<1$ and false for $\kappa \bar{d}>5$, independently of the colloidal charge. This result confirms the intuitive idea that the cell model should be valid for low values of $\kappa \bar{d}$. (It is worth mentioning that in the high (but not too high) $\kappa \bar{d}$ regime, a good alternative to the cell model is the jellium model. ${ }^{10}$ The latter supposes a fluidlike structure of the colloidal dispersion and is thus more adapted to the short-range interactions regime ${ }^{52}$ while it is equally fast and easily implemented. ${ }^{13}$ ) However, this work also demonstrates that in the intermediate $1<\kappa \bar{d}<5$ range, covering a wide set of experimental conditions, the colloidal surface charge $Z l_{\mathrm{B}} / a$ can have a dramatic effect on the error committed by the cell model. Hence, we believe that Figure 4 provides a useful reference for determining a priori if an experimental set of data may be modeled with the classical Poisson-Boltzmann cell model.

It must nonetheless be stressed that these conclusions determine conditions in which the real osmotic pressure is well approximated by its microionic contribution. It does not guarantee that the spherical cell model provides a perfect estimation of this contribution since even for low $\kappa \bar{d}$ values the cell geometry may have a significant effect on the osmotic pressure. This issue is expected to happen only for volume fractions as high as a few tenths of a percent, conditions in which other sources of discrepancy between experiments and 
purely electrostatics-based models may arise, such as hydrodynamic interactions.

\section{AUTHOR INFORMATION}

\section{Corresponding Author}

*E-mail yannick.hallez@univ-tlse3.fr.

\section{Notes}

The authors declare no competing financial interest.

\section{ACKNOWLEDGMENTS}

We warmly thank E. Trizac, C. Labbez, and A. Denton for very interesting discussions on this topic. We also thank $\mathrm{R}$. Castañeda-Priego and A. Denton for providing their respective data presented in Figure 4. This work was performed using HPC resources from CALMIP (project p1114) and GENCI (IDRIS/CINES/TGCC, grants x2013097003 and x2014097003).

\section{REFERENCES}

(1) Parsegian, V. A.; Fuller, N.; Rand, R. P. Measured work of deformation and repulsion of lecithin bilayers. Proc. Natl. Acad. Sci. U.S.A. 1979, 76, 2750-2754.

(2) Bonnet-Gonnet, C.; Belloni, L.; Cabane, B. Osmotic Pressure of Latex Dispersions. Langmuir 1994, 10, 4012-4021.

(3) Mourchid, A.; Delville, A.; Lambard, J.; Lécolier, E.; Levitz, P. Phase Diagram of Colloidal Dispersions of Anisotropic Charged Particles: Equilibrium Properties, Structure, and Rheology of Laponite Suspensions. Langmuir 1995, 11, 1942-1950.

(4) Chang, J.; Lesieur, P.; Delsanti, M.; Belloni, L.; Bonnet-Gonnet, C.; Cabane, B. Structural and thermodynamic properties of charged silica dispersions. J. Phys. Chem. 1995, 99, 15993-16001.

(5) Segad, M.; Jönsson, B.; Akesson, T.; Cabane, B. Ca/Na montmorillonite: structure, forces and swelling properties. Langmuir 2010, 26, 5782-5790.

(6) Jönsson, B.; Persello, J.; Li, J.; Cabane, B. Equation of State of Colloidal Dispersions. Langmuir 2011, 27, 6606-6614.

(7) Jin, L.; Yu, Y.-X.; Gao, G.-H. A molecular-thermodynamic model for the interactions between globular proteins in aqueous solutions: Applications to bovine serum albumin (BSA), lysozyme, a-chymotrypsin, and immuno-gamma-globulins (IgG) solutions. J. Colloid Interface Sci. 2006, 304, 77-83.

(8) Alexander, S.; Chaikin, P. M.; Grant, P.; Morales, G. J.; Pincus, P.; Hone, D. Charge renormalization, osmotic pressure, and bulk modulus of colloidal crystals: Theory. J. Chem. Phys. 1984, 80, 5776-5781.

(9) Trizac, E.; Bocquet, L.; Aubouy, M.; von Grünberg, H. H. Alexander's Prescription for Colloidal Charge Renormalization. Langmuir 2003, 19, 4027-4033.

(10) Trizac, E.; Levin, Y. Renormalized jellium model for chargestabilized colloidal suspensions. Phys. Rev. E 2004, 69, 031403.

(11) Dobnikar, J.; Castaneda-Priego, R.; von Grünberg, H.; Trizac, E. Testing the relevance of effective interaction potentials between highly-charged colloids in suspension. New J. Phys. 2006, 8, 277.

(12) Colla, T. E.; Levin, Y.; Trizac, E. A self-consistent renormalized jellium approach for calculating structural and thermodynamic properties of charge stabilized colloidal suspensions. J. Chem. Phys. 2009, 131, 074115.

(13) Falcón-González, J. M.; Castañeda Priego, R. Renormalized jellium mean-field approximation for binary mixtures of charged colloids. Phys. Rev. E 2011, 83, 041401.

(14) Marcus, R. A. Calculation of Thermodynamic Properties of Polyelectrolytes. J. Chem. Phys. 1955, 23, 1057-1068.

(15) Deserno, M.; Holm, C. In Electrostatic Effects in Soft Matter and Biophysics; Holm, C., Kékicheff, P., Podgornik, R, Eds.; NATO Science Series; Kluwer Academic Publishers: Boston, 2001.

(16) Wennerstrom, H.; Jonsson, B.; Linse, P. The cell model for polyelectrolyte systems. Exact statistical mechanical relations, Monte
Carlo simulations, and the Poisson-Boltzmann approximation. J. Chem. Phys. 1982, 76, 4665-4670.

(17) Deserno, M.; von Grünberg, H.-H. Osmotic pressure of charged colloidal suspensions: A unified approach to linearized PoissonBoltzmann theory. Phys. Rev. E 2002, 66, 011401.

(18) Lobaskin, V.; Linse, P. Simulation of an asymmetric electrolyte with charge asymmetry 60:1 using hard-sphere and soft-sphere models. J. Chem. Phys. 1999, 111, 4300-4309.

(19) Trizac, E.; Belloni, L.; Dobnikar, J.; von Grünberg, H. H.; Castañeda Priego, R. Macroion virial contribution to the osmotic pressure in charge-stabilized colloidal suspensions. Phys. Rev. E 2007, $75,011401$.

(20) Denton, A. R. PoissonBoltzmann theory of charged colloids: limits of the cell model for salty suspensions. J. Phys.: Condens. Matter 2010, 22, 364108.

(21) Das, T.; Bratko, D.; Bhuiyan, L. B.; Outhwaite, C. W. Polyelectrolyte solutions containing mixed valency ions in the cell model: A simulation and modified Poisson-Boltzmann study. J. Chem. Phys. 1997, 107, 9197-9207.

(22) Yu, Y.-X.; Wang, K.; Gao, G.-H. Density functional study on the osmotic coefficient for the DNA-electrolyte solutions. Fluid Phase Equilib. 2007, 256, 20-26.

(23) Wang, K.; Yu, Y.-X.; Gao, G.-H. Density functional study on the structural and thermodynamic properties of aqueous DNA-electrolyte solution in the framework of cell model. J. Chem. Phys. 2008, 128, 185101.

(24) Vlachy, V.; McQuarrie, D. A. A theory of cylindrical polyelectrolyte solutions. J. Chem. Phys. 1985, 83, 1927-1932.

(25) Linse, P.; Lobaskin, V. Electrostatic attraction and phase separation in solutions of like-charged colloidal particles. J. Chem. Phys. 2000, 112, 3917-3927.

(26) Rouzina, I.; Bloomfield, V. A. Macroion Attraction Due to Electrostatic Correlation between Screening Counterions. 1. Mobile Surface-Adsorbed Ions and Diffuse Ion Cloud. J. Phys. Chem. 1996, 100, 9977-9989.

(27) Sharp, K. A.; Honig, B. Calculating total electrostatic energies with the nonlinear Poisson-Boltzmann equation. J. Phys. Chem. 1990, 94, 7684-7692.

(28) Russel, W.; Saville, D.; Schowalter, W. In Colloidal Dispersions; Batchelor, G., Ed.; Cambridge University Press: Cambridge, U.K., 1999.

(29) Hoskin, N. E.; Levine, S. The Interaction of Two Identical Spherical Colloidal Particles. II. The Free Energy. Philos. Trans. R. Soc. London, Ser. A 1956, 248, 449-466.

(30) Fushiki, M. Molecular-dynamics simulations for charged colloidal dispersions. J. Chem. Phys. 1992, 97, 6700-6713.

(31) Dobnikar, J.; Chen, Y.; Rzehak, R.; von Grünberg, H. H. Manybody interactions in colloidal suspensions. J. Phys.: Condens. Matter 2003, 15, S263.

(32) Dobnikar, J.; Chen, Y.; Rzehak, R.; von Grünberg, H. H. Manybody interactions and the melting of colloidal crystals. J. Chem. Phys. 2003, 119, 4971-4985.

(33) Dobnikar, J.; Rzehak, R; von Grünberg, H. H. Effect of manybody interactions on the solid-liquid phase behavior of chargestabilized colloidal suspensions. Europhys. Lett. 2003, 61, 695.

(34) Dobnikar, J.; HaloÅ3/4an, D.; Brumen, M.; von Grünberg, H.H.; Rzehak, R. Poisson-Boltzmann Brownian dynamics of charged colloids in suspension. Comput. Phys. Commun. 2004, 159, 73-92.

(35) Dobnikar, J.; Brunner, M.; von Grünberg, H.-H.; Bechinger, C. Three-body interactions in colloidal systems. Phys. Rev. E 2004, 69, 031402.

(36) Liu, X.-D.; Fedkiw, R. P.; Kang, M. A Boundary Condition Capturing Method for Poisson's Equation on Irregular Domains. J. Comput. Phys. 2000, 160, 151-178.

(37) Lobaskin, V.; Linse, P. Accurate simulation of highly asymmetric electrolytes with charge asymmetry 20:1 and 20:2. J. Chem. Phys. 1998, 109, 3530-3541.

(38) Ermak, D. L.; McCammon, J. A. Brownian dynamics with hydrodynamic interactions. J. Chem. Phys. 1978, 69, 1352-1360. 
(39) Ohshima, H. Numerical calculation of the electrophoretic mobility of a spherical particle in a salt-free medium. J. Colloid Interface Sci. 2003, 262, 294-297.

(40) Foss, d. R.; Brady, j. F. Self-diffusion in sheared suspensions by dynamic simulation. J. Fluid Mech. 1999, 401, 243-274.

(41) Foss, D. R.; Brady, J. F. Brownian Dynamics simulation of hardsphere colloidal dispersions. J. Rheol. 2000, 44, 629-651.

(42) Fedkiw, R.; Aslam, T.; Merriman, B.; Osher, S. A non-oscillatory Eulerian approach to interfaces in multimaterial flows (the ghost fluid method). J. Comput. Phys. 1999, 152, 457-492.

(43) Gibou, F.; Fedkiw, R. P.; Cheng, L.-T.; Kang, M. A SecondOrder-Accurate Symmetric Discretization of the Poisson Equation on Irregular Domains. J. Comput. Phys. 2002, 176, 205-227.

(44) Balay, S.; Gropp, W. D.; McInnes, L. C.; Smith, B. F. Efficient Management of Parallelism in Object Oriented Numerical Software Libraries; Birkhäuser: Boston, 1997.

(45) Balay, S.; Brown, J.; Buschelman, K.; Eijkhout, V.; Gropp, W. D.; Kaushik, D.; Knepley, M. G.; McInnes, L. C.; Smith, B. F.; Zhang, H. PETSc Users' Manual U.S. Department of Energy: Washington, DC, 2010

(46) Balay, S.; Brown, J.; Buschelman, K.; Gropp, W. D.; Kaushik, D.; Knepley, M. G.; McInnes, L. C.; Smith, B. F.; Zhang, H. PETSc Web page, 2011; http://www.mcs.anl.gov/petsc.

(47) Bell, G. M.; Levine, S. Statistical thermodynamics of concentrated colloidal solutions. Part 2.-General theory of the double-layer forces on a colloidal particle. Trans. Faraday Soc. 1958, 54, 785-798.

(48) Smereka, P. The numerical approximation of a delta function with application to level set methods. J. Comput. Phys. 2006, 211, 7790.

(49) Antypov, D.; Holm, C. Optimal Cell Approach to Osmotic Properties of Finite Stiff-Chain Polyelectrolytes. Phys. Rev. Lett. 2006, 96, 088302.

(50) von Grünberg, H. H.; Belloni, L. Eccentric Poisson-Boltzmann cell model. Phys. Rev. E 2000, 62, 2493-2500.

(51) Belloni, L. Colloidal interactions. J. Phys.: Condens. Matter 2000, 12, R549.

(52) Colla, T. E.; Levin, Y. The renormalized Jellium model of colloidal suspensions with multivalent counterions. J. Chem. Phys. 2010, 133, 234105. 